Exercise-induced lactate responses in Multiple Sclerosis: A retrospective analysis Peer-reviewed author version

KEYTSMAN, Charly; HANSEN, Dominique; WENS, Inez \& OP 'T EIJNDE, Bert (2019) Exercise-induced lactate responses in Multiple Sclerosis: A retrospective analysis. In: NEUROREHABILITATION, 45(1), p. 99-106.

DOI: $10.3233 / \mathrm{NRE}-192740$

Handle: http://hdl.handle.net/1942/29957 


\title{
Exercise-induced lactate responses in Multiple Sclerosis: A retrospective analysis
}

\author{
Charly Keytsman ${ }^{\mathrm{a}, *}$, Dominique Hansen ${ }^{\mathrm{a}}$, Inez Wens ${ }^{\mathrm{a}}$ and Bert O. Eijnde ${ }^{\mathrm{b}}$ \\ ${ }^{a}$ REVAL Rehabilitation Research Center, Faculty of Rehabilitation Sciences, Hasselt University, \\ Diepenbeek, Belgium \\ ${ }^{\mathrm{b}} A D L O N$ - Sportsmedical Center, BIOMED Biomedical Research Institute, \\ Faculty of Medicine and Life Sciences, Hasselt University, Diepenbeek, Belgium
}

\begin{abstract}
.
BACKGROUND: Persons with Multiple Sclerosis have elevated resting serum lactate concentrations compared to healthy controls (HC).

OBJECTIVE: To evaluate lactate concentrations during acute exercise and/or following training in MS compared to HC.

METHODS: In this retrospective study, blood lactate concentrations ( $\mathrm{mmol} / \mathrm{l}$ ) originating from two previous studies were analyzed. Lactate concentrations originated from acute submaximal $\left(\mathrm{MS}_{\text {submax }}\right.$; $\left.\mathrm{HC}, n=11 ; \mathrm{MS}, n=32\right)$ or maximal (MS $\max$; $\mathrm{HC}, n=20$; MS, $n=24)$ exercise tests and following a 24-week mild to moderate intensity $\left(\mathrm{MS}_{\text {submax }}, n=12\right)$ or 12 -week high intensity interval $\left(\mathrm{MS}_{\max }, n=13\right)$ exercise intervention.

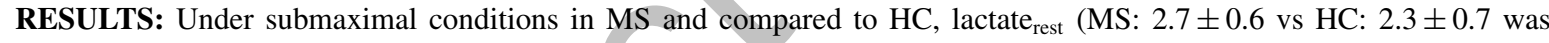
significantly $(p<0.05)$ elevated. After 24 weeks of mild-to-moderate-intensity exercise training and compared to PREvalues, lactate bout2 $(2.5 \pm 0.7$ vs $3.4 \pm 1.1)$ significantly $(p<0.05)$ decreased during submaximal testing in MS $_{\text {submax }}$. Under maximal conditions, lactate start $(2.3 \pm 1.0$ vs $1.7 \pm 0.9)$ was significantly $(p>0.05)$ elevated in MS. Twelve weeks of high intensity interval training did not improve this $(p>0.05)$.

CONCLUSIONS: Under the conditions of this retrospective analysis we conclude that lactate concentrations during acute submaximal and maximal exercise in persons with MS are similar compared to healthy controls. Moderate intensity exercise therapy appeared to improve lactate accumulation but high intensity exercise therapy did not.
\end{abstract}

Keywords: Multiple sclerosis, rehabilitation, lactate, endurance training, interval training

\section{Introduction}

In Multiple Sclerosis (MS), both central and peripheral mechanisms induce neuromuscular dysfunctions (Sharma, Kent-Braun, Mynhier, Weiner, \& Miller, 1995). Peripheral components include impaired excitation-contraction coupling and muscle energy metabolism (Sharma et al., 1995). With

*Address for correspondence: Charly Keytsman, REVAL Rehabilitation Center, Biomedical Research Institute (BIOMED), Agoralaan Building A, Hasselt University, B-3590 Diepenbeek, Belgium. Tel.: +32477/61 11 22; E-mail: charly.keytsman@uhas selt.be. respect to the latter Kent-Braun et al. (Kent-Braun et al., 1997) already demonstrated lower succinic dehydrogenase (SDH) activity and higher $\alpha$-glycerolphosphate dehydrogenase (GPDH). We have shown impaired muscle signalling for mitochondrial and myofibrillar biogenesis, with upregulated phosphoAMPK (oxidative stress) in MS (Hansen, Wens, Vandenabeele, Verboven, \& Eijnde, 2015). Furthermore, higher proportions of type II at the expense of type I fibers, together with a decrease of crosssectional area of all fibers in MS were found (Wens et al., 2014). In this population these data point towards a higher involvement of anaerobic energy 
supply. This however was not yet investigated in vivo. Interestingly, higher lactate production was recently shown by Amorini et al. (Amorini et al., 2014), showing elevated resting serum lactate concentrations in persons with MS compared to healthy controls (HC). They also demonstrated a linear correlation between serum lactate levels and the expanded disability status scale (EDSS), which might lead to new insights for objective measurable parameters to evaluate disease progression (Amorini et al., 2014).

Exercise interventions of moderate- to high intensity training have become an important part of overall rehabilitation in MS (Motl \& Gosney, 2008; Wens, Dalgas, et al., 2015). They improve muscle strength, exercise capacity and thus various functional measures (Kjolhede, Vissing, \& Dalgas, 2012), with superior effects of high intensity training compared to moderate intensity exercise (Wens, Dalgas, et al., 2015; Wens et al., 2017). However, during/following such exercise training, persons with MS report higher perceived fatigue perceptions compared to healthy controls (Wens, Dalgas, et al., 2015; Wens, Hansen, et al., 2015). Under submaximal exercise conditions lactate is typically removed by muscular gluconeogenesis and oxidation, or transported to the blood and subsequently filtered by other cells (Juel et al., 2004; Medbo, 1993). Under maximal exercise conditions, however, lactate increases rapidly as a result of anaerobic/glycolytic energy metabolism and, if not removed adequately, eventually accumulates. Lactate accumulation is then one of the contributing factors causing higher perceived fatigue (Westerblad, Allen, \& Lannergren, 2002) during exercise, which might lead to cessation of the activity. However, whether persons with MS exhibit higher lactate concentrations during exercise, possibly causing greater fatigue perceptions and perhaps poorer exercise adherence is not known.

In HC (Acevedo \& Goldfarb, 1989; Hurley et al., 1984) as well as in obese subjects (Alkahtani, King, Hills, \& Byrne, 2013) 8 to 12 weeks of suband maximal exercise training effectively improves exercise-induced lactate clearance. Whether persons with MS also profit from moderate to high intensity training (i.e. repeated exercise sessions) to gradually improve whole body lactate accumulation (Amorini et al., 2014) is not clear.

In keeping with the above lines of reasoning, the aim of the present study is to retrospectively analyze blood lactate accumulation during exercise using data that was obtained in studies previously performed by our group (Keytsman, Hansen, Wens, \& B, 2017;
Wens, Hansen, et al., 2015). We analyzed data from these studies to explore lactate accumulation during both acute (i.e. one exercise session) submaximal (Wens, Hansen, et al., 2015) and maximal (Keytsman et al., 2017) exercise bouts in persons with MS. Second, we explored whether the submaximal or maximal intensity exercise interventions applied in these studies (i.e. repeated exercise sessions) could affect whole body lactate response. We hypothesize that lactate accumulation during submaximal and maximal exercise testing is higher in persons with MS and that exercise therapy is able to decrease this.

\section{Methods}

The present study retrospectively investigated exercise-induced lactate accumulation, using lactate samples that were previously collected during submaximal (Wens, Hansen, et al., 2015) and maximal (Keytsman et al., 2017) exercise testing in MS and $\mathrm{HC}$ in two studies of our research group that were registered at ClinicalTrials.gov (NTC01718392 - NCT02466165) and published elsewhere (Keytsman et al., 2017; Wens, Hansen, et al., 2015). Data of participants of both studies were not pooled, but analysed separately (submaximal vs maximal testing/intervention) in this retrospective study.

\subsection{Participants}

In both original (Keytsman et al., 2017; Wens, Hansen, et al., 2015) studies, subjects were excluded if they were pregnant, participated in another study, had (in case of MS) an acute exacerbation 6 months prior to the start of the study or had an EDSS score $>6$. Participants were informed about the possible risk of temporary deteriorations of symptoms/disability following (sub-)maximal exercise testing (informed consent) and were asked to report this. However, during the course of these studies no such deteriorations were reported by the participants. Both studies were approved by the medical ethical committee of Hasselt University.

\subsection{Study design}

In the submaximal-study (Wens, Hansen, et al., 2015) (NTC01718392), HC $(n=11)$ and persons with MS $(n=32$, mean EDSS $3.1 \pm 0.2)$ performed a submaximal exercise test (baseline submax , Table 1) including blood lactate measurements, and a DEXA 
Table 1

Submaximal testing and exercise intervention: subject characteristics

\begin{tabular}{lcccc}
\hline & \multicolumn{2}{c}{ Baseline $_{\text {submax }}$} & & MS $_{\text {submax }}$ \\
\cline { 2 - 3 } & $\mathrm{HC}$ & $\mathrm{MS}$ & & $\mathrm{EX}$ \\
\hline Age (years) & $52.6 \pm 7.5$ & $48.1 \pm 10.4$ & & $46.3 \pm 12$ \\
Length $(\mathrm{m})$ & $1.72 \pm 9.8$ & $1.70 \pm 7.8$ & & $1.70 \pm 9.2$ \\
Weight $(\mathrm{kg})$ & $74.1 \pm 12.9$ & $73.4 \pm 14.8$ & & $72 \pm 16.1$ \\
BMI & $24.8 \pm 1.7$ & $24.4 \pm 4.62$ & & $24.6 \pm 4.5$ \\
EDSS & $/$ & $3.1 \pm 1.29$ & $3.3 \pm 1.5$ \\
$\mathrm{VO}_{2}$ bout $1(\mathrm{ml} / \mathrm{min} / \mathrm{kg})$ & $15.2 \pm 1.7$ & $13.2 \pm 2.2^{*}$ & $13.9 \pm 2.5$ \\
$\mathrm{VO}_{2}$ bout 2 $(\mathrm{ml} / \mathrm{min} / \mathrm{kg})$ & $15.2 \pm 1.8$ & $13.3 \pm 2.1^{*}$ & $14.3 \pm 2.3$ \\
\hline
\end{tabular}

Data are expressed as means \pm SD's and represent baseline subject characteristics (BMI: body mass index, EDSS: Physical Activity Scale for Individuals with Physical Disabilities, $\mathrm{VO}_{2}$ : mean oxygen uptake of the final minute of the first and second exercise bout) of healthy controls (HC, $n=11)$ and MS $(n=33)$, and a subgroup of persons with MS before (PRE) 24 weeks of mildto-moderate-intensity exercise therapy (MSsubmax, $n=12$, see methods). ${ }^{*} p<0.05$ for MS compared to HC.

scan. Hereafter, a portion of the MS group ( $\mathrm{MS}_{\text {submax }}$, $n=12$ ) was enrolled in a 24 -week mild to moderate intensity exercise intervention program. Following 24 weeks of exercise intervention, the submaximal exercise was repeated. Lactate concentrations collected in this study (Wens, Hansen, et al., 2015) were used in the present retrospective analysis to explore submaximal exercise-induced lactate accumulation.

In the maximal-study (Keytsman et al., 2017) (NCT02466165), HC $(n=20)$ and persons with MS $(n=24$, mean EDSS $2.9 \pm 0.2)$ performed a maximal exercise test (baseline $\max _{\max }$, Table 2), including blood lactate measurements, and a DEXA scan. Hereafter, a portion of the MS group ( $\mathrm{MS}_{\max }, n=13$ ) was enrolled in a 12-week high intensity interval training intervention program. Following 12 weeks of exercise intervention, the maximal exercise test was repeated. Lactate concentrations collected in this study (Keytsman et al., 2017) were used in the present retrospective analysis to explore maximal exerciseinduced lactate accumulation.

In both studies (Keytsman et al., 2017; Wens, Hansen, et al., 2015), following the baseline exercise test, $\mathrm{HC}$ and the remaining persons with MS did not further participate in the intervention programs.

\subsubsection{Allocation to exercise intervention}

In the submaximal intervention study (Wens, Hansen, et al., 2015), persons with MS were randomly allocated (matched for age, gender, EDSS) to the submaximal exercise program by sealed envelope in a 2:1 ratio (exercise or non-exercise group). The non-exercise group is not further addressed in
Table 2

Maximal testing and exercise intervention: Subject characteristics

\begin{tabular}{lcccc}
\hline & \multicolumn{2}{c}{ Baseline $_{\max }$} & & $\mathrm{MS}_{\max }$ \\
\cline { 2 - 3 } \cline { 5 - 5 } & $\mathrm{HC}$ & $\mathrm{MS}$ & $\mathrm{EX}$ \\
\hline Age (years) & $47.8 \pm 11.8$ & $50.7 \pm 8.6$ & & $51.5 \pm 7.1$ \\
Length (m) & $1.69 \pm 0.1$ & $1.70 \pm 0.1$ & & $1.71 \pm 9.1$ \\
Weight $(\mathrm{kg})$ & $69.9 \pm 14.3$ & $72.1 \pm 13.6$ & $68 \pm 12.9$ \\
BMI & $24.2 \pm 2.8$ & $24.7 \pm 4.4$ & & $23.5 \pm 3.1$ \\
EDSS & $/$ & $2.9 \pm 1.5$ & $2.6 \pm 1.5$ \\
$\mathrm{VO}_{2}$ max $(\mathrm{ml} / \mathrm{kg} / \mathrm{min})$ & $34.6 \pm 9.5$ & $24.4 \pm 7.9^{*}$ & & $28.2 \pm 8,5$ \\
\hline
\end{tabular}

Data are expressed as means \pm SD's and represent baseline subject characteristics (BMI: body mass index, EDSS: Physical Activity Scale for Individuals with Physical Disabilities, $\mathrm{VO}_{2}$ max: maximal oxygen uptake) of healthy controls (HC, $n=20)$ and persons with MS ( $n=24)$, and a subgroup of persons with MS before (PRE) 12 weeks of high intensity exercise therapy (MSmax, $n=13$, see methods). ${ }^{*} p<0.05$ for MS compared to HC.

the present retrospective study. The maximal (high intensity interval) intervention study (Keytsman et al., 2017) consisted of a pilot study, without randomization (no control group).

\subsection{Measurements}

\subsubsection{Exercise testing}

Cardiopulmonary graded exercise tests in both studies were performed on an electronically braked cycle ergometer (eBike Basic, General Electric $\mathrm{GmbH}$, Bitz, Germany). Participants were not fasted, but were instructed to minimize levels of physical activity in the preceding 24 hours prior to the exercise test. Prior to initiation of the exercise test, subjects were seated on the cycle ergometer for $3 \mathrm{~min}$ to obtain resting data.

\subsection{1 a Submaximal exercise study}

Subjects were instructed to cycle at 70rpm against a resistance corresponding to $25 \%$ (persons with MS) or $35 \%$ (HC) of predicted cycling power output $\left(\mathrm{W}_{\max }\right)$, for 6 min (first submaximal exercise bout). Hereafter, subjects remained seated on the bike for $6 \mathrm{~min}$ (recovery phase), after which a second 6 min submaximal exercise bout and a 6 min recovery phase were performed. Predicted $\mathrm{W}_{\max }$ was based on gender, age, body weight, height, and calculated by previously published formulae (Jones, Makrides, Hitchcock, Chypchar, \& McCartney, 1985). As indicated in Wens et al. 2015 (Wens, Hansen, et al., 2015), in $\mathrm{HC}$ a higher resistance was selected to obtain comparable relative exercise intensities. 


\subsection{1b Maximal graded exercise study}

Maximal exercise capacity was tested to evaluate maximal workload $\left(\mathrm{W}_{\max }\right)$, and time to exhaustion. Following a 10min standardized warm up, participants started at a low workload that gradually increased after each completed minute $\left(o^{*}\right.$ : $30 \mathrm{~W}+15 \mathrm{~W} / \mathrm{min}$, o: $20 \mathrm{~W}+10 \mathrm{~W} / \mathrm{min})$, until volitional exhaustion was reached.

\subsection{Primary outcome measure: Lactate concentrations}

Capillary blood samples in both studies were obtained to analyze blood lactate concentrations (mmol/l), using a portable lactate analyzer (Accutrend Plus, Roche Diagnostics Limited, Sussex, UK), which has previously shown to be accurate and reliable (Baldari et al., 2009). Next to absolute values and to correlate blood lactate with muscle mass, lactate concentrations were adjusted for lean tissue mass (lactate/LTM) in order to investigate lactate production per unit lean tissue mass.

\subsubsection{Submaximal lactate concentrations}

In the submaximal exercise study (Wens, Hansen, et al., 2015), immediately after the 3 min resting period $\left(\right.$ lactate $_{\text {rest }}$ ) and each $6 \mathrm{~min}$ exercise bout (lactate $_{\text {bout } 1}$, lactate bout2), capillary blood samples were obtained from the fingertip to analyze blood lactate concentrations.

\subsubsection{Maximal lactate concentrations}

In the maximal exercise study (Keytsman et al., 2017), no resting lactate concentrations were collected. Immediately after the first exercise minute (lactate $_{\text {start }}$ ) and subsequently every $2 \mathrm{~min}$ until volitional cessation (lactate max $_{\text {), capillary blood samples }}$ were taken from the earlobe to analyze blood lactate concentrations.

\subsection{Secondary outcome measures}

\subsubsection{Cardiorespiratory outcomes}

During the exercise test, oxygen uptake $\left(\mathrm{VO}_{2}\right.$ $\mathrm{ml} / \mathrm{min}$ ) was assessed breath-by-breath, after which this data were averaged every 10s (Jaeger Oxycon, Erich Jaeger GmbH, Germany).

In the submaximal (Wens, Hansen, et al., 2015) exercise study, mean oxygen uptake $\left(\mathrm{VO}_{2} \mathrm{ml} / \mathrm{min} / \mathrm{kg}\right)$ of the final minute (steady state) of the first $\left(\mathrm{VO}_{2}\right.$ bout 1$)$ and second $\left(\mathrm{VO}_{2}\right.$ bout 2 ) exercise bout were reported.
In the maximal (Keytsman et al., 2017) exercise study, oxygen uptake at the level of maximal exhaustion $\left(\mathrm{VO}_{2} \mathrm{ml} / \mathrm{min} / \mathrm{kg}\right)$ was reported.

\subsubsection{Body composition}

Lean tissue mass in both studies was obtained using Dual Energy X-ray Absorptiometry scan (DEXA) (Hologic Series Delphi-A Fan Beam X-ray Bone Densitometer, Vilvoorde, Belgium). A calibrated analogue weight scale (Seca $\left.{ }^{\circledR}\right)$ was used to measure total body mass.

\subsection{Exercise intervention programs}

\subsubsection{Submaximal exercise study}

Part of the persons with MS ( $\left.\mathrm{MS}_{\text {submax }}\right)$ that were tested submaximally participated in a supervised 24week combined training program, at a frequency of 5 sessions per two weeks. Each session started with a cardiovascular part, consisting of cycling and treadmill walking or running (Technogym $\left.{ }^{\circledR}\right)$. Session duration and intensity increased as the program proceeded, starting from $1 \times 6 \mathrm{~min} / \mathrm{session}$ to $3 \times 10$ $\mathrm{min} /$ session, interspersed by $2 \mathrm{~min}$ of rest. The second part consisted of resistance training (leg press, leg curl, leg extension, vertical traction, arm curl, and chest press; Technogym $\left.{ }^{\circledR}\right)$. To improve muscle fitness, sets of repetitions gradually increased during intervention, from $1 \times 10$ repetitions to $4 \times 15$ repetitions, with maximal attainable load, interspersed by $2 \mathrm{~min}$ of rest. All exercises were performed at a mild to moderate workload corresponding to 12-14 ratings of perceived exertion (RPE) on a 20-point Borg scale.

\subsubsection{Maximal exercise study}

Part of the persons with MS ( $\left.\mathrm{MS}_{\max }\right)$ that were exercise tested maximally performed a 12-week HIIT training program on a cycle ergometer and moderate-to-high intensity resistance training under close supervision. During the first 6-week training cycle, exercise duration gradually increased from $5 \times 1 \mathrm{~min}$ ( $1 \mathrm{~min}$ rest intervals) to $5 \times 2 \mathrm{~min}$. Exercise intensity was $100 \%$ of the maximal workload (highest power output at the point of exhaustion obtained during the exercise test), corresponding to $85-90 \%$ of the maximal heart rate. During the second 6-week training cycle, duration remained stable at $5 \times 2 \mathrm{~min}$ and the workload (Watt) increased to $100 \%$ of maximal heart rate. The second part consisted of intensity resistance training. Here, training intensity and volume were adjusted throughout 
the intervention program from $1 \times 10$ repetitions to $2 \times 20$ repetitions at a maximal attainable load.

Prior to the first training session, a familiarization visit was performed to determine starting loads for the resistance exercises. Because persons with MS often experience bilateral strength differences between legs (Thoumie, Lamotte, Cantalloube, Faucher, \& Amarenco, 2005), exercise training was performed at similar relative workloads. Consequently, resistance training of the lower limbs was performed unilaterally during both training interventions.

\subsection{Statistical analysis}

All data were analysed using SPSS v.22.0 (IBM). Normality was analysed using Shapiro-Wilk test. Baseline differences between $\mathrm{HC}$ and MS were analyzed using the Mann Whitney $\mathrm{U}$ test (unpaired). Pre-post differences within the MS exercise groups were analyzed using Wilcoxon Signed Ranks tests (paired). All data are presented as means \pm SD's and the threshold for statistical significance was set at $p<0.05$.

\section{Results}

\subsection{Subject characteristics}

During submaximal testing in MS, oxygen uptake of the first $\left(\mathrm{VO}_{2}\right.$ bout $1: 13.2 \pm 2.2$ vs $15.2 \pm 1.7$ $\mathrm{ml} / \mathrm{min} / \mathrm{kg})$ and second bout $\left(\mathrm{VO}_{2}\right.$ bout $2: 13.3 \pm 2.1$ vs $15.2 \pm 1.8 \mathrm{ml} / \mathrm{min} / \mathrm{kg}$ ) were significantly lower compared to $\mathrm{HC}(p=0.003$ and $p=0.003$ respectively). During maximal exercise testing in MS, maximal oxygen uptake $\left(\mathrm{VO}_{2}\right.$ max: $24.4 \pm 7.9$ vs $34.6 \pm 9.5 \mathrm{ml} / \mathrm{min} / \mathrm{kg}$ ) was significantly lower compared to HC $(p<0.0001)$. Other baseline characteristics, during sub- and maximal testing, did not differ between HC and MS (Table $1 \& 2$ ).

\subsection{Lactate concentrations}

\subsubsection{Submaximal lactate concentrations}

In MS, lactate rest $(+17 \%)$ was significantly elevated compared to $\mathrm{HC}$, whereas lactate bout 1 and lactate $_{\text {bout2 }}$ did not differ between groups $(p>0.05)$. When adjusted for lean tissue mass, similar effects were noted for lactate rest $_{\text {LTM }}(+33 \%$ compared to HC) and lactate bout $1_{1} /$ LTM (no difference with HC). Compared to $\mathrm{HC}$, the second submaximal exercise bout induced higher lactate bout $2_{2} /$ LTM $(+33 \%)$
Table 3

Blood lactate concentrations during submaximal and maximal exercise testing

\begin{tabular}{lcc}
\hline & HC & MS \\
\hline Submax. exercise test & & \\
Lactate $_{\text {rest }}$ & $2.3 \pm 0.7$ & $2.7 \pm 0.6^{*}$ \\
Lactate $_{\text {bout } 1}$ & $3.1 \pm 1.2$ & $3.1 \pm 0.8$ \\
Lactate $_{\text {bout } 2}$ & $2.7 \pm 1.5$ & $3.0 \pm 0.7$ \\
Lactate $_{\text {rest }} /$ LTM & $5.2 \pm 1.9$ & $6.9 \pm 2.1^{*}$ \\
Lactate $_{\text {bout } 1} /$ LTM & $6.7 \pm 2.4$ & $7.9 \pm 2.7$ \\
Lactate $_{\text {bout } 2 / L T M}$ & $5.8 \pm 2.8$ & $7.7 \pm 2.5^{*}$ \\
Max. exercise test $_{\text {Lactate }_{\text {start }}}$ & & \\
Lactate $_{\text {max }}$ & $1.7 \pm 0.9$ & $2.3 \pm 1.0^{*}$ \\
Lactate $_{\text {start }} /$ LTM & $5.6 \pm 1.7$ & $5.4 \pm 1.8$ \\
Lactate $_{\text {max }} /$ LTM & $3.9 \pm 2.6$ & $5.5 \pm 2.9^{*}$ \\
Data $_{\text {are }}^{\text {expressed }}$ & $12.5 \pm 3.7$ & $13.5 \pm 5.9$ \\
\hline
\end{tabular}

Data are expressed as means \pm SD's and represent both baseline blood lactate concentrations $(\mathrm{mmol} / \mathrm{L})$ and lactate concentrations adjusted for lean tissue mass (LTM, [lactate/LTM]*100) of healthy controls (HC) and persons with MS during sub-

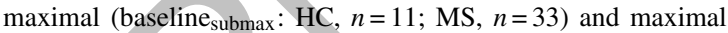

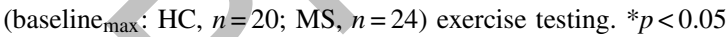
compared to baseline $\mathrm{HC}$.

in MS (Table 3). Following 24 weeks of mild-tomoderate-intensity exercise training and compared to PRE exercise intervention values lactate rest $(2.6 \pm 0.4$ $\mathrm{mmol} / \mathrm{L}$ vs. $2.2 \pm 0.7 \mathrm{mmol} / \mathrm{L}, \quad p=0.1 ; 95 \% \mathrm{CI}$ $-0.1-0.9)$ and lactate bout $1(3.3 \pm 0.7 \mathrm{mmol} / \mathrm{L}$ vs. $2.8 \pm 0.9 \mathrm{mmol} / \mathrm{L}, p=0.07 ; 95 \% \mathrm{CI}-0.5-0.9)$ tended to decrease. Twenty four weeks of submaximal exercise significantly $(p<0.05)$ reduced blood lactate during the second exercise bout (95\% CI 0.3-1.4, Table 4). When corrected for lean tissue mass, similar results were obtained $(95 \% \mathrm{CI} 0.00-0.03$ ).

\subsubsection{Maximal lactate concentrations}

Compared to $\mathrm{HC}$, lactate start $(+35 \%)$ and lactate $_{\text {start }} /$ LTM $(+41 \%)$ was significantly elevated in MS, whereas lactate max $_{\text {ax }}$ and lactate max $_{\max } /$ LTM did not differ between groups (Table 3). Twelve weeks of high intensity interval training did not improve this in $\mathrm{MS}_{\max }$ (Table 4).

\section{Discussion}

This retrospective study confirms higher resting blood lactate levels in MS compared to healthy controls. However, during acute submaximal and maximal exercise, these differences in blood lactate concentrations disappear. Twenty-four weeks of moderate intensity exercise therapy tended to improve resting blood lactate levels and reduced blood lactate during submaximal exercise. Surpris- 
Table 4

Exercise test blood lactate concentrations following exercise therapy

\begin{tabular}{lccc}
\hline & $\begin{array}{c}\text { PRE } \\
\text { MS }\end{array}$ & $\begin{array}{c}\text { POST } \\
\text { MS }\end{array}$ & $p$-value \\
& & & \\
MS $_{\text {submax }}$ & & & \\
Lactate $_{\text {rest }}$ & $2.6 \pm 0.4$ & $2.2 \pm 0.7$ & 0.10 \\
Lactate $_{\text {out } 1}$ & $3.3 \pm 0.7$ & $2.8 \pm 0.9$ & 0.07 \\
Lactate $_{\text {bout } 2}$ & $3.4 \pm 1.1$ & $2.5 \pm 0.7 *$ & 0.00 \\
Lactate $_{\text {est }} /$ LTM & $6.6 \pm 1.9$ & $5.5 \pm 2.3$ & 0.06 \\
Lactate $_{\text {bout } 1} /$ LTM & $8.2 \pm 2.2$ & $6.9 \pm 2.2$ & 0.05 \\
Lactate $_{\text {bout }} /$ LTM & $8.4 \pm 2.7$ & $6.3 \pm 2.1 *$ & 0.01 \\
MS $_{\text {max }}$ & & & \\
Lactate $_{\text {start }}$ & $2.2 \pm 1.1$ & $2.1 \pm 0.7$ & 0.71 \\
Lactate $_{\text {max }}$ & $5.8 \pm 1.7$ & $5.5 \pm 1.3$ & 0.50 \\
Lactate $_{\text {start }} /$ LTM & $5.9 \pm 3.8$ & $5 \pm 2.6$ & 0.51 \\
Lactate $_{\text {max }} /$ LTM & $14.9 \pm 6.7$ & $13.3 \pm 4.8$ & 0.37 \\
\hline Data $_{\text {at }}$ expsed &
\end{tabular}

Data are expressed as means \pm SD's and represent blood lactate concentrations $(\mathrm{mmol} / \mathrm{L})$ and lactate concentrations adjusted for lean tissue mass (LTM, [lactate/LTM]*100) of a subgroup of persons with MS before (PRE) and after (POST) 24 weeks of mildto-moderate-intensity exercise (MSsubmax: $n=12$ ) or 12 weeks of high intensity exercise therapy (MSmax: $n=13$ ) respectively. $* p<0.05$ compared to corresponding PRE MS.

ingly, 12 weeks of high intensity exercise therapy in this population did not have comparable effects during maximal testing.

We here confirm increased resting/start blood lactate levels in persons with MS compared to HC as recently reported by Amorini et al. (Amorini et al., 2014). Amorini and coworkers were the first to show elevated resting serum lactate concentrations in MS and they assumed that this originated from either neural or muscular tissue. To further explore this, we used a submaximal and maximal exercise test to induce muscle-based blood lactate accumulation. With the exception of the second submaximal exercise bout, the exercise-induced lactate responses during both acute submaximal and maximal exercise were similar in MS and HC. Therefore, increased subjective fatigue perceptions during exercise in persons with MS (Wens, Dalgas, et al., 2015; Wens, Hansen, et al., 2015) does not seem to originate from higher lactate concentrations.

In HC (Acevedo \& Goldfarb, 1989; Hurley et al., 1984) and other populations (Alkahtani et al., 2013) exercise training at sub- or maximal intensity is known to effectively reduce exercise-induced lactate accumulation. In the present study, a 24-week moderate intensity exercise intervention tended to reduce resting blood lactate levels and blood lactate following a first bout of submaximal effort. Following a second bout of aerobic muscle work blood lactate was effectively lower. Twelve weeks of high intense exercise therapy did not have such effect on both resting and exercise-induced blood lactate concentrations either or not corrected for lean tissue mass. This may suggest an abnormal lactate response in MS under anaerobic/glycolytic circumstances such as during high intensity training which confirms earlier reported skeletal muscle anaerobic metabolism malfunctions in MS (Hansen et al., 2015; Kent-Braun et al., 1997; Sharma et al., 1995). High intensity exercise therapy in MS, however, elicits superior effects for exercise capacity, muscle contractile characteristics (Wens, Dalgas, et al., 2015) and glucose tolerance (Wens et al., 2017) compared to moderate intensity training. As such, a combination of high intense exercise training with moderate intensity physical therapy seems ideal to maximally benefit from the advantages of both training modalities.

It is important to note that an exercise test performed under both submaximal and maximal conditions following moderate and after high-intensity exercise training would be ideal. Unfortunately, the available retrospective data did not allow this. Therefore, and because of the different lactate responses during moderate and high intensity training exercise presented in this study, future research seems warranted.

Finally, the results of this retrospective study may implicate that muscular activation under both submaximal and maximal exercise conditions might be a valid strategy to reduce elevated resting lactate concentrations in MS. Furthermore, any strategy that may improve exercise-induced lactate response during high intensity exercise therapy may be worthwhile investigating, since this may improve exercise adherence and performance and thus possibly lead to better clinical outcomes in MS.

\subsection{Limitations}

In the present study, resting values of the submaximal exercise testing in both $\mathrm{HC}$ and MS (2.3 vs 2.7 $\mathrm{mmol} / \mathrm{l}$ respectively) are higher than usual. During the maximal graded exercise test this can be explained by the fact that the first blood sample (lactate start $_{\text {) was }}$ taken after $1 \mathrm{~min}$ of exercise and therefor does not resemble full resting conditions. Under the conditions of the submaximal test (lactate rest $_{\text {) }}$ it is more difficult to explain but possibly could be attributed to the fact that the seated resting position prior to the submaximal test simply was too short to obtain actual resting blood lactate. Furthermore, the different time-points 
of baseline lactate values ('resting' vs 'start' lactate), during the submaximal and maximal exercise conditions makes it more difficult to interpret this data. Therefore, future research should include adequate resting phases prior to the lactate measurements, as well as comparable/similar measurements of resting lactate concentrations. Moreover, a fasting period of $10-12 \mathrm{~h}$ prior to the exercise testing procedures may be preferred in future research, as this may impact the data regarding resting lactate values. The present study does not include data regarding power analysis because the analysis of lactate concentrations was based on retrospective data from two previous studies of our research group (Keytsman et al., 2017; Wens, Hansen, et al., 2015). Therefore, future research regarding resting and exercise-induced lactate concentrations in MS should be powered in order to draw solid conclusions. Furthermore, possible bias for exercise testing/intervention may also include different MS phenotypes and levels of cardiorespiratory fitness, as well as instructions/descriptions of activities performed before the testing procedures. Despite the fact that in the present retrospective study, we intended for the first time to explore exercise-induced lactate concentrations in MS, we recommend future research to include the above described limitations.

\section{Conclusion}

Under the conditions of the present retrospective study we conclude that persons with MS indeed have higher resting blood lactate concentrations. During acute submaximal and maximal exercise, however, lactate responses appear to be similar between MS and healthy controls. Furthermore, moderate intensity exercise therapy probably improves lactate concentrations but high intensity exercise therapy did not.

\section{Conflict of interest}

The authors declare no conflicts of interest.

\section{References}

Acevedo, E. O., \& Goldfarb, A. H. (1989). Increased training intensity effects on plasma lactate, ventilatory threshold, and endurance. Med Sci Sports Exerc, 21(5), 563-568.
Alkahtani, S. A., King, N. A., Hills, A. P., \& Byrne, N. M. (2013). Effect of interval training intensity on fat oxidation, blood lactate and the rate of perceived exertion in obese men. Springerplus, 2, 532. doi:10.1186/2193-1801-2-532

Amorini, A. M., Nociti, V., Petzold, A., Gasperini, C., Quartuccio, E., Lazzarino, G.,... Tavazzi, B. (2014). Serum lactate as a novel potential biomarker in multiple sclerosis. Biochim Biophys Acta, 1842(7), 1137-1143. doi:10.1016/j.bbadis.2014.04.005

Baldari, C., Bonavolonta, V., Emerenziani, G. P., Gallotta, M. C., Silva, A. J., \& Guidetti, L. (2009). Accuracy, reliability, linearity of Accutrend and Lactate Pro versus EBIO plus analyzer. Eur J Appl Physiol, 107(1), 105-111. doi:10.1007/s00421-0091107-5

Hansen, D., Wens, I., Vandenabeele, F., Verboven, K., \& Eijnde, B. O. (2015). Altered signaling for mitochondrial and myofibrillar biogenesis in skeletal muscles of patients with multiple sclerosis. Transl Res, 166(1), 70-79. doi:10.1016/j.trsl.2015.01.006

Hurley, B. F., Hagberg, J. M., Allen, W. K., Seals, D. R., Young, J. C., Cuddihee, R. W., \& Holloszy, J. O. (1984). Effect of training on blood lactate levels during submaximal exercise. $J$ Appl Physiol Respir Environ Exerc Physiol, 56(5), 1260-1264.

Jones, N. L., Makrides, L., Hitchcock, C., Chypchar, T., \& McCartney, N. (1985). Normal standards for an incremental progressive cycle ergometer test. Am Rev Respir Dis, 131(5), 700-708. doi:10.1164/arrd.1985.131.5.700

Juel, C., Klarskov, C., Nielsen, J. J., Krustrup, P., Mohr, M., \& Bangsbo, J. (2004). Effect of high-intensity intermittent training on lactate and $H+$ release from human skeletal muscle. Am J Physiol Endocrinol Metab, 286(2), E245-251. doi:10.1152/ajpendo.00303.2003

Kent-Braun, J. A., Ng, A. V., Castro, M., Weiner, M. W., Gelinas, D., Dudley, G. A., \& Miller, R. G. (1997). Strength, skeletal muscle composition, and enzyme activity in multiple sclerosis. J Appl Physiol (1985), 83(6), 1998-2004.

Keytsman, C., Hansen, D., Wens, I., \& B, O. E. (2017). Impact of high-intensity concurrent training on cardiovascular risk factors in persons with multiple sclerosis - pilot study. Disabil Rehabil, 1-6. doi:10.1080/09638288.2017.1395086

Kjolhede, T., Vissing, K., \& Dalgas, U. (2012). Multiple sclerosis and progressive resistance training: A systematic review. Mult Scler, 18(9), 1215-1228. doi:10.1177/1352458512437418

Medbo, J. I. (1993). Glycogen breakdown and lactate accumulation during high-intensity cycling. Acta Physiol Scand, 149(1), 8589. doi:10.1111/j.1748-1716.1993.tb09595.x

Motl, R. W., \& Gosney, J. L. (2008). Effect of exercise training on quality of life in multiple sclerosis: A meta-analysis. Mult Scler, 14(1), 129-135. doi:10.1177/1352458507080464

Sharma, K. R., Kent-Braun, J., Mynhier, M. A., Weiner, M. W., \& Miller, R. G. (1995). Evidence of an abnormal intramuscular component of fatigue in multiple sclerosis. Muscle Nerve, 18(12), 1403-1411. doi:10.1002/mus.880181210

Thoumie, P., Lamotte, D., Cantalloube, S., Faucher, M., \& Amarenco, G. (2005). Motor determinants of gait in 100 ambulatory patients with multiple sclerosis. Mult Scler, 11(4), 485-491.

Wens, I., Dalgas, U., Vandenabeele, F., Grevendonk, L., Verboven, K., Hansen, D., \& Eijnde, B. O. (2015). High Intensity Exercise in Multiple Sclerosis: Effects on Muscle Contractile Characteristics and Exercise Capacity, a Randomised Controlled Trial. PLoS One, 10(9), e0133697. doi:10.1371/journal.pone.0133697 
Wens, I., Dalgas, U., Vandenabeele, F., Krekels, M., Grevendonk, L., \& Eijnde, B. O. (2014). Multiple sclerosis affects skeletal muscle characteristics. PLoS One, 9(9), e108158. doi:10.1371/journal.pone.0108158

Wens, I., Dalgas, U., Vandenabeele, F., Verboven, K., Hansen, D., Deckx, N.,... Eijnde, B. O. (2017). High Intensity Aerobic and Resistance Exercise Can Improve Glucose Tolerance in Persons With Multiple Sclerosis: A Randomized Controlled Trial. Am J Phys Med Rehabil, 96(3), 161-166. doi:10.1097/PHM.0000000000000563
Wens, I., Hansen, D., Verboven, K., Deckx, N., Kosten, L., Stevens, A. L.,... Eijnde, B. O. (2015). Impact of 24 Weeks of Resistance and Endurance Exercise on Glucose Tolerance in Persons with Multiple Sclerosis. Am J Phys Med Rehabil. doi: $10.1097 / \mathrm{phm} .0000000000000257$

Westerblad, H., Allen, D. G., \& Lannergren, J. (2002). Muscle fatigue: Lactic acid or inorganic phosphate the major cause? News Physiol Sci, 17, 17-21. 